\title{
Modeling and simulation of the impact behavior of soft polymeric-foam-based back protectors for winter sports
}

\author{
Stefano Signettia ${ }^{\mathrm{a}, 1}$, Marco Nicotra ${ }^{\mathrm{b}}$, Martino Colonna ${ }^{\mathrm{b}, *}$, Nicola M. Pugno $^{\mathrm{a}, \mathrm{c}, \mathrm{d}, *}$ \\ ${ }^{a}$ Laboratory of Bio-Inspired and Graphene Nanomechanics, \\ Department of Civil, Environmental and Mechanical Engineering, University of Trento, \\ via Mesiano 77, I-38123 Trento, Italy \\ ${ }^{b}$ Department of Civil, Chemistry, Environmental and Materials Engineering, \\ University of Bologna, via Terracini 28, I-40122 Bologna, Italy \\ ${ }^{c}$ School of Engineering and Materials Science, Queen Mary University of London, \\ Mile End Road, E1 4NS London, UK \\ ${ }^{d}$ Ket-Lab, Edoardo Amaldi Foundation, Italian Space Agency, via del Politecnico snc, I-00133 Roma, Italy
}

\begin{abstract}
Objectives: Winter sports are high-energy outdoor activities involving high velocities and acrobatic maneuvers, thus raising safety concerns. Specific studies on the impact mechanics of back protectors are very limited. In this study analytical and numerical models are developed to rationalize results of impact experiments and propose new design procedures for this kind of equipment.
\end{abstract}

Design: Different soft-shell solutions currently available on the market are compared. In particular, the role of dynamic material constitutive properties and of environmental temperature (which affects mainly material stiffness) on energy absorption capability are evaluated.

Methods: Starting from dynamic mechanical-thermal characterization of the closed-cell polymeric foams constituting the protectors, we exploited analytical modeling and Finite Element Method simulations to interpret experimental data from drop weight impact test and to characterize protectors at different temperatures and after multiple impacts.

Results: The temperature and frequency dependent properties of these materials characterize their impact behavior. Modeling results are in good agreement with impact tests. Results

\footnotetext{
${ }^{*}$ Corresponding author.

Email addresses: martino.colonna@unibo.it (Martino Colonna), nicola.pugno@unitn.it (Nicola M. Pugno)

${ }^{1}$ Present address: Department of Mechanical Engineering, Korea Advanced Institute of Science and Technology (KAIST), 291 Daehak-ro, Yuseong-gu, Daejeon 34141, Republic of Korea
} 
demonstrate how ergonomic soft-shell solution provides an advantage with respect to traditional hard-shell in terms of impact protection. Moreover, it can maintain nearly unaltered its protective properties after multiple impacts on the same point.

Conclusions: The coupled analytical-simulation approach here presented could be extensively used to predict the impact behavior of such equipment, starting from material characterization, allowing to save costs and time for physical prototyping and tests for design and optimization.

Keywords: Back protectors, Winter sports, Back injuries, Soft polymeric foams, Impact testing, FEM modeling

\section{Introduction}

Winter sports are performed by an estimate of $200 \mathrm{M}$ people in the word, including different ages and skill groups [1]. This number is in constant growth, also thanks to increasing popularity in new Asian markets, pushed by recent PyeongChang 2018 and future Beijing 2022 Winter Olympic Games. Winter sports, especially alpine skiing and snowboard, are generally high-energy outdoor activities involving high velocity, jumps and acrobatic maneuvers and the inherent risks, coupled with an increasing congestion on ski slopes, raise serious safety concerns. Nowadays, traumatic injuries occur with a rate up to about 3 per 1000 skier-days and up to about 8 per 1000 snowboarder-days [1, 2] and, also due to the high healthcare expenses connected with these injuries, there is a strong interest in prevention. The statistics of the injuries distribution over the body have discording results depending on the country taken into exam [1 4]. Nevertheless, all these studies agree that the most affected areas are head, shoulders, spine and knees. In particular, a Swiss study reports that back injuries are more common in snowboarding with respect to skiing (18.3\% vs. 10.2\%) [5]. Moreover, snowboarders sustain 4-5.7 spinal injuries per 100000 days [6]. Risk reduction can be pursued at different levels, from regulation of ski activities and risk-awareness [7] to the development of more efficient individual protective equipment, such as helmets [8, 9] and back protectors [10, 11] or external passive system, such as safety barriers [12]. 
Historically, all the back protectors had a hard-shell construction consisting of a hard outer shell of thermoplastic material (e.g., polypropylene) with an inner soft padding foam and some textiles, forming the lining. In these products the shock attenuation relies on the distribution of the impact force over a wider area by the outer rigid material, also resistant to abrasive and puncture injuries. The main collateral disadvantage of this solution is the bad air flow which causes excessive sweating and poor thermal comfort during activity [13]. Also the ergonomics is highly limited, since the rigidity does not allow complete freedom of movements and may lead to compression of the zones in contact with the body, resulting in pain or incorrect body movements. To overcome these problems, an increasing number of products are based on the new soft-shell technology, recently proposed by manufactures, which adopts soft polymeric foams. In this solution the protection is given by energy dissipation through reversible deformation of cell walls [14. Moreover, the pseudo-dilatant nature of the polymeric foams ensures an adaptive behavior, reacting like hard and rigid materials when subjected to high deformation rate, enabling a high level of protection, and like soft viscous materials at service load condition [14], providing good flexibility and comfort during movements. Their higher comfort arises also from their excellent thermal characteristics, since the production processes and the material properties allow to obtain perforated breathable structures. Usually the protective elements are enclosed in a high strength fabric vest which adheres perfectly to the body and retains the correct position of the protector element during crash, ensuring its effectiveness. A pseudo-dilatant behavior can be also obtained by the employment of auxetic foams where the negative Poisson's ratio causes a local increase of density under the impact area due to induced compressive stresses. These solutions have already been demonstrated to perform better with respect to the traditional counterparts [15].

Despite the peculiarity of ski back protectors, there is no specific performance standard related to snow sports. Companies are currently borrowing motorcycling standards [16, 17] to test impact performances, design, and market their products. However, their adequacy has already been questioned [18]. Drop weight impact testing [19] is a common technique to assess the shock absorbing properties and has been applied in different fields (e.g., sports, defense, health care) and classes of materials. Dynamic Mechanical Thermal Analysis (DMTA) [20 22] 
is acknowledged in the field to correlate material properties and impact performances, also accounting for aging effects [23]. This method consists in applying an oscillatory force to a beam sample and analyzing its viscoelastic frequency-dependent mechanical response. DMTA is of relevant importance since this kind of equipment is subjected to large temperature changes during use and storage. A limited influence of temperature on the visco-elastic properties is desirable in a material for ski back protectors allowing a constant performance in different scenarios, both in terms of impact absorption and ergonomics. By the way, the usage statistics and specific studies on the mechanics of back protectors are very limited [4, 11, 18] and generally mechanical studies are limited to experimental performance assessment without an engineering optimization of the product. While several works exploited both analytical and numerical modeling to assess the impact protection of motorcycle helmets [24, 25], there is no analogous research, up to the best of authors' knowledge, applied to back protectors for winter sports and addressing specific needs for practitioners.

Following a previous experimental work by the authors on commercial protectors [26], we here rationalize the obtained results by finite element method (FEM) impact simulation and analytical modeling to compare different soft-shell solutions currently available on the market. The role of the constitutive behavior, environmental temperature, and multiple impact on the energy absorption capability is evaluated. A characterization procedure is proposed and a simulation tool is developed for the design and optimization of such equipments.

\section{Methods}

\subsection{Impact testing}

Impact tests have been performed using an Instron Dynatup $9250 \mathrm{HV}$ drop weight (gravity driven) impact testing machine using a flat circular impact head with a diameter of $4.5 \mathrm{~cm}$. The sample is supported by a flat aluminum anvil which reproduces the real scenario where the protector adheres to the skier's back. The basic assembly is described alsewhere [19]. To avoid the influence of the curvature of the protectors the impacts have been performed only on flat sections at a distance of at least $5 \mathrm{~cm}$ from the edge of the protectors. The samples have been tested at $+20^{\circ} \mathrm{C}$ and after being kept at $-5^{\circ} \mathrm{C}$ for 24 hours. The total testing time 
was below 30 seconds, so it can be assumed that the samples maintained their temperature during the tests. All the samples were impacted using a mass of $5 \mathrm{~kg}$ dropped from a height of about $1 \mathrm{~m}$, to ensure an impact energy of $50 \mathrm{~J}$. Sample deflection, impact force and velocity were computed with a sampling rate of $600 \mathrm{~Hz}$. This type of tests provides a more complete information set on the material properties compared to the EN 1621-2 standard [17], which only requires measure of the transmitted force.

\subsection{Analytical dynamic model}

To describe the impact process in the drop weight configuration we recall the solution to the problem of a perfectly rigid flat punch in frictionless contact with a semi-infinite elastic solid. Under the hypothesis that mechanical vibrations can be neglected -and this is the case of soft materials- the impact event between two colliding bodies can be described by the following differential equation:

$$
m \ddot{w}(t)+c \dot{w}(t)+k w(t)=0,
$$

where $w(t)$ is the displacement of the substrate at the center of the impact contact area (hence equal to the displacement of the impactor, assuming it as rigid), $m=\frac{m_{1} m_{2}}{m_{1}+m_{2}}$ with $m_{1}$ and $m_{2}$ being the mass of the impactor and of the substrate respectively, $c$ is the coefficient of viscous damping, and $k=2 E R /\left(1-\nu^{2}\right)$ is the contact stiffness of the substrate in case of flat punch impact [27], with $R$ being the radius of the impactoror, $E$ is the Young's modulus of the deformable substrate, and $\nu$ its Poisson's ratio. Note that in our case $m_{2} \rightarrow \infty$ and thus $m=m_{1}$, since the protector is supported by a rigid and fixed substrate. Hence, Equation (1) represents a single degree of freedom (SDOF) damped harmonic oscillator. The integration of Equation (1) with initial condition $\dot{w}(0)=v_{0}$ and $w(0)=0$ yields to the following relation:

$$
w(t)=\frac{v_{0}}{\omega_{\mathrm{D}}} \mathrm{e}^{-\xi \omega t} \sin \omega_{\mathrm{D}} t
$$

where $v_{0}$ is the initial impact velocity, $\xi=c /(2 \sqrt{\mathrm{km}})$ is the ratio between the damping coefficient $c$ and its critical value, $\omega=\sqrt{k / m}$ is the pulse, and $\omega_{\mathrm{D}}=\omega \sqrt{\left(1-\xi^{2}\right)}$ is the damped pulse. The value of damping coefficient to be used in both analytical and FEM models can be related to the phase angle measured from the DMTA analysis as [28]:

$$
c=\frac{k_{\mathrm{b}}}{\bar{\omega}} \tan \delta
$$


where $\bar{\omega}=2 \pi \bar{f}$, with $\bar{f}$ being the imposed oscillation frequency of DMTA analysis and $k_{\mathrm{b}}=3 E J / l^{3}$ is the bending stiffness of the cantilever samples used in the DMTA analysis (see Supplementary Section S1.3). Computed values of $\xi$ are reported in Supplementary Table S4.

The maximum average impact pressure $\bar{\sigma}_{\max }$ within the substrate occurs at the instant of zero relative velocity $(\dot{w}=0)$, thus at a time:

$$
\tau=\frac{2}{\omega_{\mathrm{D}}} \arctan \left[-\frac{\xi}{\sqrt{1-\xi^{2}}}+\sqrt{1+\left(\frac{\xi}{\sqrt{1-\xi^{2}}}\right)^{2}}\right]
$$

which, consistently, is inversely proportional to the ratio $k / m$ showing how softer materials can increase the time-to-peak $\tau$. From Equation (4) it is evident how this particular formulation is valid for subcritical damping consition only $(\xi<1)$ and this is the case of the material tested in this work (see Supplementary Table S4). Finally, by inserting the value of the time-to-peak obtained by Equation (4) into Equation (2) it is possible to derive the maximum deflection $w_{\text {peak }}$ and force $F_{\text {peak }}$. The corresponding mean contact pressure is:

$$
\bar{\sigma}_{\max }=\frac{2 E w(\tau)}{\pi R\left(1-\nu^{2}\right)}
$$

\subsection{Finite Element model}

FEM simulations were performed to analyze and complement the experimental results. A rigid cylindrical impactor of radius $R=2.25 \mathrm{~cm}$ and mass $m=5 \mathrm{~kg}$ hits a deformable target at a impact velocity $v_{0}=4.47 \mathrm{~m} / \mathrm{s}$, hence replicating exactly the setup of the the drop weight test. The protector is represented by a cylindrical plate of radius $100 \mathrm{~mm}$ supported at the bottom (fixed boundary condition) to reproduce the experimental configuration. Only a quarter of the plate was modeled due to the symmetry of the system by setting proper boundary conditions (see Supplementary Figure S3). Thickness, density and material properties were changed case by case according to the values obtained by the characterization of protectors (see density and thickness reported in Supplementary Table S1 and DMTA-derived properties at different temperatures reported in Supplementary Table S4). The used material properties refer to DMTA analysis operated at a characteristic frequency of $50 \mathrm{~Hz}$. This frequency was the highest that could be reach by our instrumentation and it was demonstrated to properly characterize the material properties to model the specific impact regime (energy and strain 
rate) tested in the experiments. The material model used for the polymeric protector is a constitutive law specifically developed for low density, closed cell foams [29]. This constitutive theory accounts both the elastic and inelastic responses of rigid polyurethane foams by decomposing the foam behavior into two parts: a skeleton and a nonlinear elastic continuum in parallel. The skeleton accounts for the foam behavior in the elastic and plateau regimes. The nonlinear elastic continuum accounts for the lock-up of the foam due to internal gas pressure and cell-wall interactions. Both the impactor and the substrate are modeled with hexahedral under-integrated solid elements. Spurious deformation modes (hourglass) were properly controlled and the related energy was monitored and verified to not affect simulation results. Two-way penalty based contact is implemented between the impactor and the target and friction is cautelatively neglected in the model. The numerical models were implemented and solved within the explicit finite element solver ABAQUS. Additional modeling details are reported in the Supplementary Material (Section S2).

\section{Results and discussion}

\subsection{Protector testing and thermal effects}

The results of the force-displacement curves obtained from impact test at $+20^{\circ} \mathrm{C}$ are reported in Figure 1,a. In general, a good shock absorbing material should present a low impact force spread over a longer time, resulting in a reduced impulse and thus to a smaller probability of injury. In this regard protector 1, 2, and 4 have similar behavior while protector 3 shows sensibly higher impact force and low time-to-peak. Note that, since the specific setup of the test, the absorbed energy (area under the force-deflection curve) is the same for all protectors and equal to the initial impactor kinetic energy $K_{0}$ but the protectors differ from each other in the way they dissipate this energy. All protectors are able to sustain the impact without damage as the applied impact energy is below the Level 1 protection level to which all samples are certified. The force-displacement curves of all protectors have similar characteristics, typical for this kind of materials [30]: a first linear elastic region, controlled

by cell walls bending and stretching, is followed by a deformation plateau, controlled by non-linear elastic buckling of the cell walls. These two regions can be clearly distinguished 
Table 1: Comparison of characteristic impact properties among all methods used in this analysis for tests at $+20^{\circ} \mathrm{C}$ and $-5^{\circ} \mathrm{C}$.

\begin{tabular}{ccccccccccc} 
& & \multicolumn{3}{c}{ Experiments } & \multicolumn{3}{c}{ FEM Simulations } & \multicolumn{3}{c}{ Analytical model } \\
\cline { 3 - 10 } $\mathbf{T}$ & Protector & $F_{\text {peak }}$ & $\tau$ & $\bar{\sigma}_{\text {max }}$ & $F_{\text {peak }}$ & $\tau$ & $\bar{\sigma}_{\text {max }}$ & $F_{\text {peak }}$ & $\tau$ & $\bar{\sigma}_{\text {max }}$ \\
& & {$[\mathrm{kN}]$} & {$[\mathrm{ms}]$} & {$[\mathrm{MPa}]$} & {$[\mathrm{kN}]$} & {$[\mathrm{ms}]$} & {$[\mathrm{MPa}]$} & {$[\mathrm{kN}]$} & {$[\mathrm{ms}]$} & {$[\mathrm{MPa}]$} \\
\hline & & 5.30 & 4.8 & 3.33 & 5.58 & 4.7 & 3.51 & 4.08 & 5.2 & 2.57 \\
& 2 & 5.73 & 4.8 & 3.60 & 6.40 & 4.6 & 4.02 & 4.17 & 4.8 & 2.62 \\
& 3 & 8.64 & 4.3 & 5.43 & 9.30 & 4.2 & 5.85 & 7.93 & 4.6 & 4.99 \\
& 4 & 5.55 & 4.3 & 3.49 & 5.87 & 4.3 & 3.69 & 4.87 & 4.7 & 3.06 \\
\hline$-5{ }^{\circ} \mathrm{C}$ & 1 & 6.29 & 2.6 & 3.95 & 6.10 & 2.8 & 3.84 & 6.38 & 2.9 & 4.01 \\
& 2 & 11.20 & 1.7 & 7.04 & 10.80 & 1.8 & 6.79 & 10.21 & 2.3 & 6.42 \\
& 3 & 5.22 & 2.9 & 3.28 & 5.31 & 2.8 & 3.34 & 5.05 & 2.5 & 3.18 \\
& 4 & 15.53 & 0.8 & 9.76 & 15.38 & 0.8 & 9.67 & 14.83 & 1.6 & 9.35 \\
\hline
\end{tabular}

by a "yield" point. Finally, the force increases sharply due to the densification of the foam whose stiffness tends to the one of the bulk material. Experimental curves are compared to the ones obtained by the FEM simulations. Results by different methods in terms of peak force $F_{\text {peak }}$, time-to-peak $\tau$ and mean impact pressure at peak force $\bar{\sigma}_{\max }$ are summarized in Table 1 showing good agreement between all methods of analysis.

Complementary results at $-5^{\circ} \mathrm{C}$ are reported in Figure 1.b. At low temperature all the soft-shell protectors present an increase of the curve slope (hard behavior) with respect to the behavior at $+20^{\circ} \mathrm{C}$, since the material is more rigid due to the reduced motions of polymer segments, resulting in an increase of the apparent stiffness and yield point. Protectors 2 and 4 show the largest increase of the peak impact force and shortening of the time-to-peak (Table 1). This result can be directly imputed to the highest thermal sensitivity showed in the material stiffness (Supplementary Section S1.3 and Table S4) and thus the effectiveness of this kind of protector should be thoroughly investigated because of its lower performance at lower temperatures, with a behavior more similar to the hard-shell protectors, i.e. high impact force spread in a short time. Thus, on the basis of impact analysis at different temperatures 

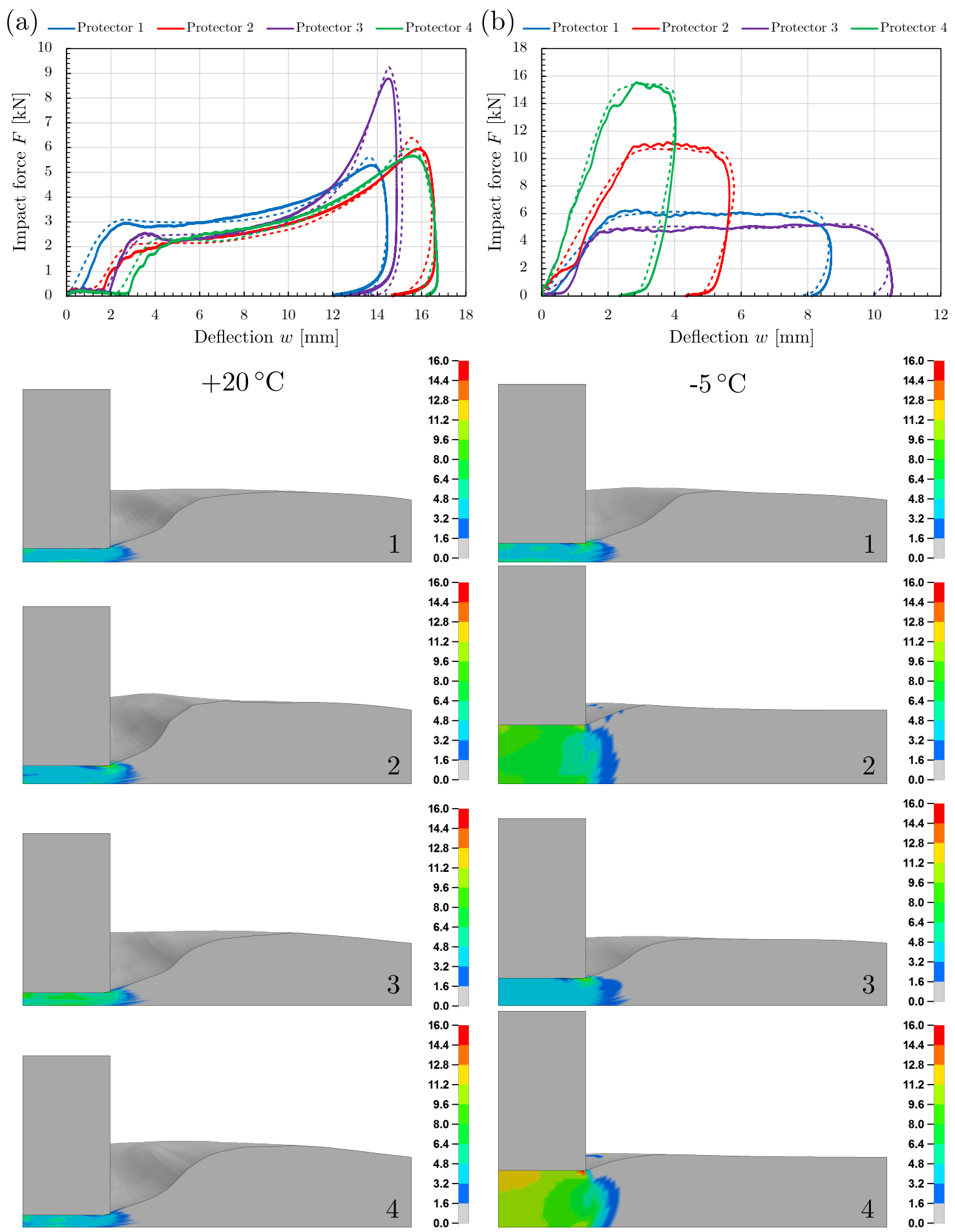

Figure 1: Experimental (continuous) and FEM (dashed) force-deflection curves for the four tested protectors at (a) $+20^{\circ} \mathrm{C}$ and (b) $-5^{\circ} \mathrm{C}$. In the bottom panels the snapshots from FEM simulations at the characteristic impact time $(t=\tau$, refer to values in Table 1) are depicted with contour plot of impact pressure (units in $\mathrm{MPa}$ ). Stress field can be compared to the experimentally derived and analytically predicted stresses reported in in Table 1 . 
protector 1 seems to be the preferable solution among the all tested to reduce the severity of the injury after a fall. In this sense, soft-shell protectors differ from hard-shell technology which does not show a significant change at low temperature since the mechanism of impact protection does not rely on viscous damping, almost negligible, but on material stiffness [26], which is not significantly affected in those kind of materials. FEM snapshots of Figure 1.a-b show how the stiffening of the material at low temperature yields to lower deflection and distributes the load over a wider area with respect to the same protectors analyzed at room temperature. Characteristic results from all performed analyses at $-5{ }^{\circ} \mathrm{C}$ are reported and compared in Table 1 .

\subsection{Multi-impact performance}

The behavior of protector 2 has been tested at $+20^{\circ} \mathrm{C}$ under multiple impact by repeating the drop weight test five times on the same area, with an interval of 1 minute between tests. Figure 2 shows the force-displacement curves of the 5 impact events under the same conditions. It is evident the increase in $w_{\text {peak }}$ and a reduction of the yielding force prior to the plateau. The explanation of this behavior can be connected to the damage that occurs in the foam structure after each impact event, which leads to a softening of the material [30]. However, at high deformation, an increase in the peak impact force $(+23.5 \%)$ is observed. This behavior, apparently in contrast with the softening of these materials, can be explained by the fact that the occurred damage in the material enhances the non-linearity of the constitutive response, yielding higher elastic modulus at higher compressive strain, since the accumulated permanent deformation yields to a progressively denser material. Secondly, the increase of $F_{\text {peak }}$ may be attributed to the fact that the higher deflection makes the impactor to feel more the interaction of the rigid substrate. This should not be accounted as a test artifact since it represents the real scenario offered by the skier's back. Therefore, a compromise between material properties and thickness (ergonomics) must be properly evaluated as well as the degradation of properties after several impacts. However, it must be noted that the increase in the impact force after 5 events is much limited with respect to hard-shell protectors which have proven to be less sensible to temperature but have poor multi-impact capabilities, , being prone to progressive damage [26]. 


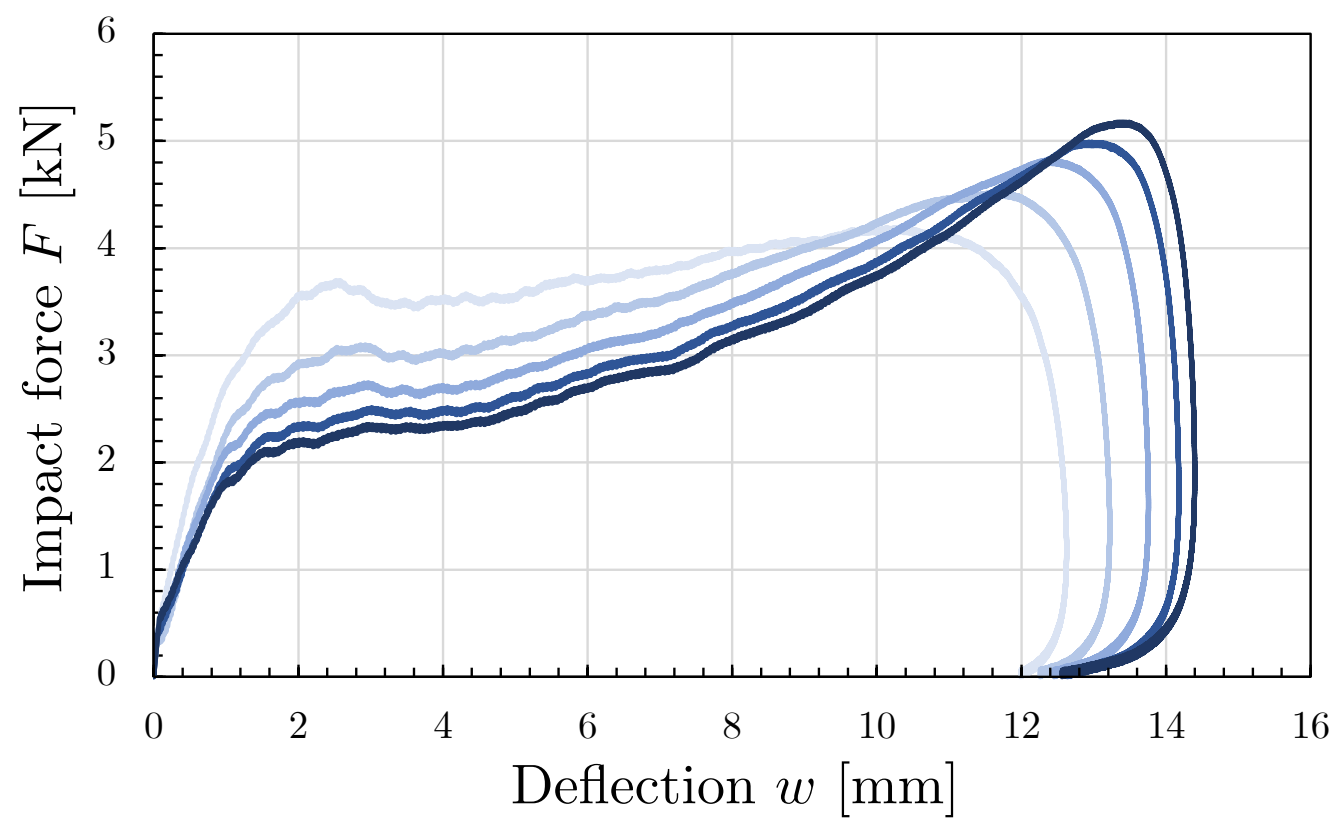

Impact 1 - Impact 2 Impact 3 -Impact 4 Impact 5

Figure 2: Experimental force-deflection curves for protector 2 under multiple impact at $+20^{\circ} \mathrm{C}$.

\section{Conclusions}

The study of the thermo-mechanical and impact properties of materials used for soft-shell back protectors showed their strain-rate-sensitive behavior. Indeed, the visco-elastic properties, mainly elastic modulus and damping coefficient, depend on the frequency of the applied stress. These protectors are more rigid at high speed impacts (high-frequency load) while are softer for low strain rates, resulting in a good ergonomic comfort during during natural movements but protecting the body in case of a collision. Results on some commercially available back protectors show that some products are very sensitive to temperature, and in the real environmental can lead to a significant increase (up to about 2-3 times) of the impact force. In this sense, polymeric foams with low temperature dependence should be preferred. The high sensitivity to temperature with respect to traditional rigid protectors is counterbalanced by a better multi-impact behavior, which makes soft-shells preferable. The developed FEM impact model is able to reproduce the experimentally observed behavior for the different protector, and can give additional information regarding the deformation and stress states that could be of help for future advanced design and optimization of such 
equipments. The procedure presented in this paper can be used as a protocol during the design of body protectors and ski helmets internal pads in order to select the best performing materials and geometries, thus reducing cost and time of the development process. Future investigations should include a wider range of scenarios -limited in this work- accounting different impact energies/velocities, impactors of different shapes (also simulating cutting and high penetrating objects) and angle of incidence, and different internal structures of the foam [31, 32]. Moreover, a more thoroughly understanding of the behavior of these materials in a wider temperature range is necessary as well as a deeper correlation between material characterization by DMTA and actual impact conditions for better prediction capability of models.

\section{Practical implications}

- The analytical and numerical models presented here can predict with good reliability the impact behavior of polymeric-foam-based protectors. These methods could represent a viable alternative for manufacturers to save in physical prototyping and experiments during the design stage, especially for optimization studies.

- More real and specific impact scenarios can be included in the models, overcoming the limits of current standardized test and classification by protection levels, which are borrowed from motorcycling standards. Tailored design of protectors, e.g. with zoned properties, according to specific needs of different sport activities is an example.

- The results presented here can provide guidelines for future studies and development of standards dedicated to winter sports protectors.

\section{Acknowledgements}

NMP is supported by the European Commission under the Graphene Flagship Core 2 (WP14 "Composites", no. 785219) and the FET Proactive ("Neurofibers", no. 732344) and by the Italian Ministry of Education, University and Research (MIUR) under the "Departments of Excellence" grant L. 232/2016. SS acknowledges financial support from Ermenegildo Zegna Founder's Scholarship 2017-2018. 


\section{References}

[1] Ackery A, Hagel BE, Provvidenza C, et al. An international review of head and spinal cord injuries in alpine skiing and snowboarding. Inj Prev 2007;13(6):368-75. doi:10.1136/ip.2007.017285.

[2] McBeth PB, Ball CG, Mulloy RH, et al. Alpine ski and snowboarding traumatic injuries: incidence, injury patterns and risk factors for 10 years. Am J Surg 2008;197(5):560-4. doi:10.1016/j . amj surg.2008.12.016.

[3] Levy AS, Hawkes AP, Hemminger LM, et al. An Analysis of Head Injuries among Skiers and Snowboarders. J Trauma Acute Care 2002;53(4):695-704. doi:10.1097/01 TA.0000032121.91608.1B.

[4] Burtscher M, Gatterer H, Flatz M, et al. Effects of Modern Ski Equipment on the Overall Injury Rate and the Pattern of Injury Location in Alpine Skiing. Clin J of Sport Med 2008;18(4):355-7. doi:10.1097/MJT.0b013e31815fd0fe.

[5] bfu - Swiss Council for Accident Prevention . Accidents - Report nr. 71. 2017. URL: https://www.bfu.ch/en/research-and-statistics/statistics.

[6] Yamakawa H, Murase S, Sakai H, et al. Spinal Injuries in Snowboarders: Risk of Jumping as an Integral Part of Snowboarding. J Trauma Acute Care 2001;50(6):11015. URL: https://journals.lww.com/jtrauma/pages/articleviewer.aspx?year= 2001\&issue $=06000 \&$ article $=00020 \&$ type $=$ abstract .

[7] Hildebrandt C, Mildner E, Hotter B, et al. Accident prevention on ski slopes - Perceptions of safety and knowledge of existing rules. Accident Anal Prev 2011;45(4):352. doi:10. $1136 / \mathrm{bjsm} .2011 .084038 .119$.

[8] Jung CS, Zweckberger K, Schick U, et al. Helmet use in winter sport activities - attitude and opinion of neurosurgeons and non-traumatic-brain- injury-educated persons. Acta Neurochirur 2011;153(1):101-6. doi:10.1007/s00701-010-0704-8. 
[9] Russel K, Christie J, Hagel BE. The effect of helmets on the risk of head and neck injuries among skiers and snowboarders: a meta-analysis. Can Med Assoc J 2010;182(4):333-40. doi:10.1503/cmaj.091080.

[10] Engsberg JR, Standeven JW, Shurtleff TL, et al. Spinal cord and brain injury protection: testing concept for a protective device. Spinal Cord 2009;47(8):634-9. doi:10.1038/sc. 2009.1.

[11] Michel FI, Schmitt KU, Liechti B, et al. Functionality of back protectors in snow sports concerning safety requirements. Procedia Engineer 2010;2(2):2869-74. doi:10.1016/j proeng.2010.04.080.

[12] Petrone N. The Effect of Impact Speed, Construction, and Layout of Different Ski Safety Barriers on Peak Decelerations and Penetration Values of a Solid Dummy During Full Scale Impacts. In: Johnson R, Shealy J, Greenwald R, et al., editors. Ski Trauma and Safety; vol. 19. ASTM International. ISBN 978-0-8031-7539-6; 2012, p. 153-70. doi:10.1520/STP104509.

[13] Dotti F, Ferri A, Moncalero M, et al. Thermo-physiological comfort of soft-shell back protectors under controlled environmental conditions. Appl Ergon 2016;56:144-52. doi:10.1016/j.apergo.2016.04.002.

[14] Palmer RM, Green PC. Energy absorbing material. 2008. URL: https://patents. google.com/patent/US7794827B2/en; US 7381460 B2.

[15] Foster L, Peketi P, Allen T, et al. Application of Auxetic Foam in Sports Helmets. Appl Sci-Basel 2018;8(3):354. doi:10.3390/app8030354.

[16] EN 1621-1, Motorcyclists' protective clothing against mechanical impact. Requirements and test methods for impact protectors. 2012.

[17] EN 1621-2, Motorcyclists' protective clothing against mechanical impact. Motorcyclists back protectors. Requirements and test methods. 2003. 
[18] Schmitt KU, Liechti B, Michel FI, et al. Are current back protectors suitable to prevent spinal injury in recreational snowboarders? Brit J Sport Med 2010;44(11):822-6. doi:10.1136/bjsm.2010.072728.

[19] ISO 6603-2, Determination of puncture impact behaviour of rigid plastics. 2000.

[20] ASTM D4065-12, Standard Practice for Plastics: Dynamic Mechanical Properties: Determination and Report of Procedures. 2012.

[21] Rodríguez-Pérez MA, Velasco JI, Arencón D, et al. Mechanical characterization of closed-cell polyolefin foams. J Appl Polym Sci 2000;75(1):156-66. doi:10.1002/(SICI) 1097-4628(20000103) 75:1<156: :AID-APP18>3.0.CO;2-U.

[22] Rodríguez-Pérez MA. Effect of Chemical Composition, Density and Cellular Structure on the Dynamic Mechanical Response of Polyolefin Foams. Cell Polym 2002;21(1):117-36. doi:10.1177/026248930202100202.

[23] Verdejo R, Mills JN. Performance of EVA foam in running shoes. In: Ujihashi S, Haake SJ, editors. The Engineering of Sport; vol. 4. Blackwell Science. ISBN 0-632-06481-1; 2002, p. $580-7$.

[24] Kostopoulos V, Markopoulos YP, Giannopoulos G, et al. Finite element analysis of impact damage response of composite motorcycle safety helmets. Compos Part B-Eng 2002;33(2):99-07. doi 10.1016/S1359-8368(01)00066-X.

[25] Nasim M, Brasca M, Khosroshahi SF, et al. Understanding the impact properties of polymeric sandwich structures used for motorcyclists' back protectors. Polym Test 2017;61:249-57. doi:10.1016/j.polymertesting.2017.05.025.

[26] Nicotra M, Moncalero M, Messori M, et al. Thermo-mechanical and impact properties of polymeric foams used for snow sports protective equipment. Procedia Engineer 2014;72:678-83. doi:10.1016/j jproeng.2014.06.115. 
[27] Harding JW, Sneddon IN. The elastic stresses produced by the indentation of the plane surface of a semi-infinite elastic solid by a rigid punch. Math Proc Cambridge 1945;41(1):16-26. doi 10.1017/S0305004100022325.

[28] Graesser EJ, Wong CR. The Relationship of Traditional Damping Measures for Materials with High Damping Capacity. 1991. URL: www.dtic.mil/dtic/tr/fulltext/u2/ a235347.pdf.

[29] Neilsen MK, R. D Krieg HL, Schreyer . A constitutive theory for rigid polyurethane foam. Polym Eng Sci 1995;35(5):387-94. doi:10.1002/pen.760350503.

[30] Klempner D, Sendijarevic V. Handbook of polymeric foams and foam technology. 2nd ed.; Hanser Publishers; 2004.

[31] Signetti S, Pugno NM. Evidence of optimal interfaces in bio-inspired ceramic-composite panels for superior ballistic protection. J Eur Ceram Soc 2014;34(11):2823-31. doi:10. $1016 / \mathrm{j} \cdot$ jeurceramsoc.2013.12.039.

[32] Chen Q, Shi Q, Signetti S, et al. Plastic collapse of cylindrical shell-plate periodic honeycombs under uniaxial compression: experimental and numerical analyses. Int $\mathrm{J}$ of Mech Sci 2016;111:125-33. doi:10.1016/j.ijmecsci.2016.03.020. 


\section{-Supplementary Material-}

\section{Modeling and simulation of the impact behavior of soft polymeric-foam-based back protectors for winter sports}

Stefano Signetti, ${ }^{\dagger} \|$ Marco Nicotra, ${ }^{\ddagger}$ Martino Colonna, ${ }^{*, \ddagger}$ and Nicola M. Pugno*, $\uparrow, \uparrow, \S$

$\dagger$ Laboratory of Bio-Inspired and Graphene Nanomechanics, Department of Civil, Environmental and Mechanical Engineering, University of Trento, via Mesiano 77, I-38123 Trento, Italy

$\ddagger$ Department of Civil, Chemistry, Environmental and Materials Engineering, University of Bologna, via Terracini 28, I-40122 Bologna, Italy

IS School of Engineering and Materials Science, Queen Mary University of London, Mile End Road, E1 4NS London, UK

$\S$ Ket-Lab, Edoardo Amaldi Foundation, Italian Space Agency, via del Politecnico snc, I-00133 Roma, Italy

||Present address: Department of Mechanical Engineering, Korea Advanced Institute of Science and Technology (KAIST), 291 Daehak-ro, Yuseong-gu, Daejeon, 34141 Republic of Korea

E-mail: martino.colonna@unibo.it; nicola.pugno@unitn.it 


\section{S1 Experimental materials and methods}

\section{S1.1 Materials}

Four commercial back protectors (Figure S1), already tested in a precedent work by the authors, ${ }^{1}$ have been used for model validation. All the samples are of size L (no modification was performed) and are based on the soft-shell technology. According to the manufacturers, protectors 1, 2, and 4 have a certified protection Level 2, while protector 3 is a Level $1 .{ }^{2}$ Table S1 reports the characteristics of protectors. Hardness measurements were performed using a Hildebrand Shore A durometer at $+23^{\circ} \mathrm{C}$ according to ISO 868 standard ${ }^{3}$ specifications. In this method, a Shore A indenter is forced into the sample and the depth of the penetration after 15 seconds is measured. The hardness is inversely proportional to the penetration and directly correlated to the elastic modulus of the material. Die-cutted samples with size $10 x 10 \mathrm{~cm}^{2}$ were extracted from each protector, maintaining the original thickness. For each sample a total of five measurements were preformed calculating the mean values.
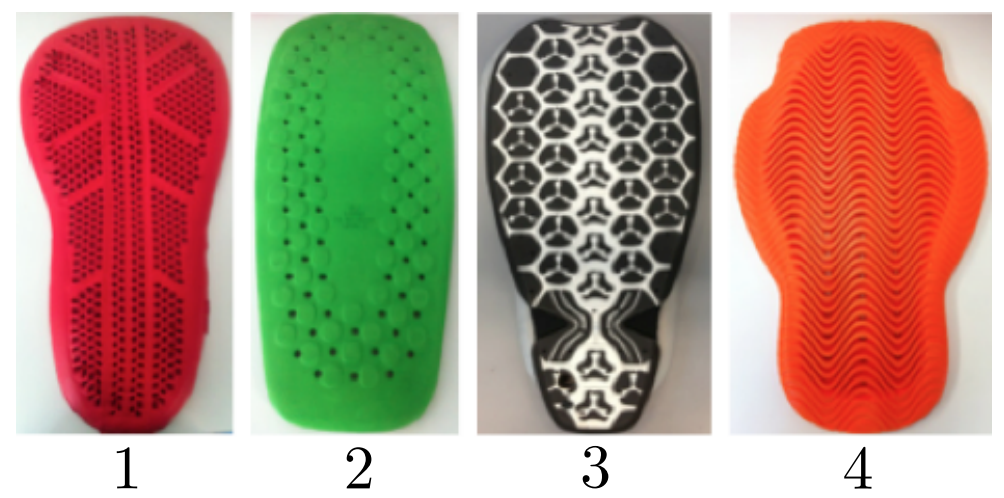

Figure S1: The four commercial back protectors tested and used for model verification. ${ }^{1}$

Table S1: Characteristics of the four back protectors tested. ${ }^{1}$

\begin{tabular}{cccccc}
\hline Protector & $\begin{array}{c}\text { Protection } \\
\text { level }\end{array}$ & $\begin{array}{c}\text { Mass } \\
{[\mathrm{g}]}\end{array}$ & $\begin{array}{c}\text { Density } \\
{\left[\mathrm{g} / \mathrm{cm}^{3}\right]}\end{array}$ & $\begin{array}{c}\text { Thickness } \\
{[\mathrm{mm}]}\end{array}$ & $\begin{array}{c}\text { Hardness } \\
\text { Shore A }\end{array}$ \\
\hline 1 & 2 & 283 & 0.15 & 16 & 33 \\
2 & 2 & 657 & 0.35 & 19 & 23 \\
3 & 1 & 325 & 0.22 & 16 & 15 \\
4 & 2 & 472 & 0.35 & 18 & 14 \\
\hline
\end{tabular}




\section{S1.2 Chemical composition and microstructure}

The chemical composition of the materials used for the protectors has been determined by Fourier Transform Infrared Spectroscopy (FT-IR) with a Perkin Elmer Spectrum One instrument, using an Attenuated Total Reflectance (ATR) detector. Each spectrum is the result of 32 scans with a resolution of $4 \mathrm{~cm}^{-1}$. Wavelength range varies between $4000 \mathrm{~cm}^{-1}$ and $650 \mathrm{~cm}^{-1}$. The comparison with a database of polymeric foams shows that protectors 1,2 and 3 are made of a blend of polyvinyl acetate, ethylene vinyl acetate (EVA) and nitrile butadiene rubber. Protector 4 is made of a polyurethane blend containing polydimethylsiloxane.

The samples were examined using a Nova NanoSEM 450 scanning electron microscope in order to characterize the internal morphology, chemical composition, crystalline structure and orientation. Cross-sections of the foams were obtained by fracturing the protectors at low temperature, after immersion in liquid nitrogen. From an analysis of the SEM images it has been possible to determine the microstructure of the soft-shell protectors. A qualitative analysis of the micrograph reveals a closed cell structure for all the protectors, with cell dimensions and wall thickness that depend on the density and chemical composition of the foams (Figure S2). 


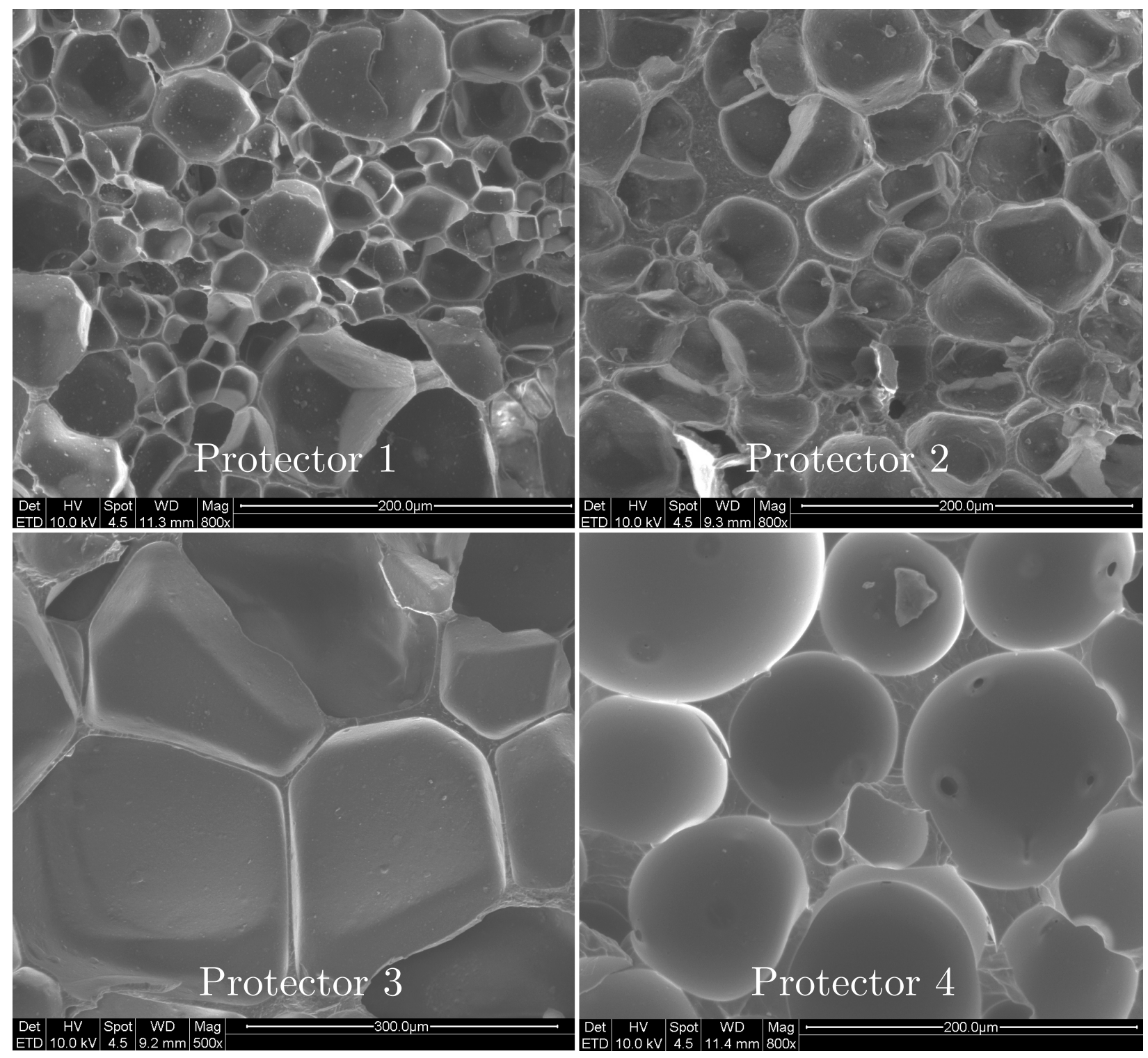

Figure S2: Scanning Electron Microscope (SEM) images of the four soft-shell foam protectors. Protector 1: non-homogeneous cell dimension, varying between $19 \mu \mathrm{m}$ and $90 \mu \mathrm{m}$, and thin cell walls throughout the whole sample. Protector 2: homogeneous cell dimension, around $57 \mu \mathrm{m}$, and thick cell walls throughout the whole sample. Protector 3: homogeneous cell dimension, around $230 \mu \mathrm{m}$, and medium sized cell walls throughout the whole sample. Protector 4: spherical cells with radius of about $80 \mu \mathrm{m}$ and variable wall thickness. 


\section{S1.3 Dynamic Mechanical Thermal Analysis (DMTA)}

DMTA tests ${ }^{4-6}$ were performed with a Rheometrics DMTA 3E model, bending a cantilever sample within a strain $\varepsilon=0.1 \%$. The samples with dimensions $20 \times 8 \times 4 \mathrm{~mm}$ were die-cut directly from the protectors. In order to obtain a complete understanding of the materials properties and their variation with frequency and temperature, three frequencies were used $(\bar{f}=1 \mathrm{~Hz}, 10 \mathrm{~Hz}$, and $50 \mathrm{~Hz})$ in a temperature range between $-40{ }^{\circ} \mathrm{C}$ and $+30^{\circ} \mathrm{C}$.

Due to the visco-elastic nature of the polymers tested, a sinusoidal stress path induces a sinusoidal stress response with the same frequency but out of phase by an angle $\delta$ :

$$
\left\{\begin{array}{l}
\sigma=\sigma_{0} \cos (\bar{\omega} t)=\sigma_{0} e^{i \bar{\omega} t} \\
\varepsilon=\varepsilon_{0} \cos (\bar{\omega} t-\delta)=\varepsilon_{0} e^{-i \delta} e^{i \bar{\omega} t}
\end{array}\right.
$$

where $\bar{\omega}=2 \pi \bar{f}$ is the pulse of the hysteresis cycle; $\sigma_{0}$ and $\varepsilon_{0}$ are respectively the amplitude of stress and strain in the cycle. The ratio $\sigma / \varepsilon$, namely the material Young's modulus, can be rewritten as a complex number:

$$
\frac{\sigma}{\varepsilon}=\frac{\sigma_{0}}{\varepsilon_{0}}(\cos \delta+i \sin \delta)=E^{\prime}+i E^{\prime \prime}=E(1+i \tan \delta),
$$

where $E^{\prime}$ is the elastic stiffness of the material (storage modulus, equivalent to Young's modulus for low damping) while $E^{\prime \prime}$, and consequently $\tan \delta=\frac{E^{\prime \prime}}{E^{\prime}}$, is a measure of the material damping (loss modulus) and can be related to the energy dissipated in form of heat due to molecular rearrangements and internal friction.

The results of the DMTA analysis with respect to environmental temperature are reported in Table S2. ${ }^{1}$ All samples show the typical behavior of polymeric materials with decrease of the elastic modulus with increasing temperature. The elastic modulus of protector 1 has the smallest variation in the temperature range investigated with only $2.3 \%$ decrease. Protector 4 , instead, is the most affected by temperature, having the highest elastic modulus at low temperature and the lowest at high temperature. This kind of behavior is not desirable in 
a material used in winter sport applications. Data on the influence of temperature on the damping behavior $(\tan \delta)$ are also reported (Table S2). All samples show a qualitative similar behavior with temperature. The influence of load frequency on the values of the elastic modulus $E^{\prime}$ and the $\tan \delta$ for the different materials at $+20^{\circ} \mathrm{C}$ are reported in Table S3. In a similar way as temperature, also the frequency $\bar{f}$ of the applied stress plays an important role on the performance of the final product. All protectors show an increase of the elastic modulus when increasing the frequency. For example, a 8-fold increase can be observed for protector 4 moving from $1 \mathrm{~Hz}$ to $50 \mathrm{~Hz}$. This frequency-sensitive pseudo-dilatant behavior is responsible for the particular properties of soft foams used for protective equipment. The materials used for soft-shell protectors have high tan $\delta$ values ranging, at $1 \mathrm{~Hz}$, from 0.32 to 1.2, indicating a strong damping behavior for all tested materials. The $\tan \delta$ values decrease by increasing the frequency of the applied stress. More detailed results showing the combined effect of temperature and load frequency, continuous scan from $-40{ }^{\circ} \mathrm{C}$ to $30{ }^{\circ} \mathrm{C}$ with an applied frequency of $1 \mathrm{~Hz}$ and heating rate of $3^{\circ} \mathrm{C} / \mathrm{min}$, can be found elsewhere. ${ }^{1}$

Table S2: Effect of the temperature on elastic modulus $E^{\prime}$ and phase angle $\tan \delta$ measured at $1 \mathrm{~Hz}$.

\begin{tabular}{ccccccc}
\hline \multirow{2}{*}{ Protector } & \multicolumn{3}{c}{$E^{\prime}[\mathrm{Pa}]$} & & \multicolumn{3}{c}{$\tan \delta$} \\
& $-40{ }^{\circ} \mathrm{C}$ & $0^{\circ} \mathrm{C}$ & $+30^{\circ} \mathrm{C}$ & $-40^{\circ} \mathrm{C}$ & $0^{\circ} \mathrm{C}$ & $+30^{\circ} \mathrm{C}$ \\
\hline 1 & $1.4 \cdot 10^{7}$ & $9.1 \cdot 10^{6}$ & $7.8 \cdot 10^{5}$ & 0.02 & 0.09 & 0.49 \\
2 & $4.4 \cdot 10^{7}$ & $2.0 \cdot 10^{7}$ & $2.3 \cdot 10^{5}$ & 0.03 & 0.19 & 1.80 \\
3 & $3.2 \cdot 10^{7}$ & $1.9 \cdot 10^{7}$ & $4.1 \cdot 10^{5}$ & 0.05 & 0.12 & 1.02 \\
4 & $7.6 \cdot 10^{7}$ & $4.5 \cdot 10^{7}$ & $1.7 \cdot 10^{5}$ & 0.02 & 0.14 & 1.80 \\
\hline
\end{tabular}

Table S3: Effect of the testing frequency on elastic modulus $E^{\prime}$ and phase angle $\tan \delta$ at $+20^{\circ} \mathrm{C}$.

\begin{tabular}{ccccccccc}
\hline \multirow{2}{*}{ Frequency } & \multicolumn{2}{c}{ Protector $\mathbf{1}$} & \multicolumn{2}{c}{ Protector 2 } & Protector 3 & \multicolumn{2}{c}{ Protector 4 } \\
\cline { 2 - 8 } & $\begin{array}{c}E^{\prime} \\
{[\mathrm{MPa}]}\end{array}$ & $\tan \delta$ & $\begin{array}{c}E^{\prime} \\
{[\mathrm{MPa}]}\end{array}$ & $\tan \delta$ & $\begin{array}{c}E^{\prime} \\
{[\mathrm{MPa}]}\end{array}$ & $\tan \delta$ & $\begin{array}{c}E^{\prime} \\
{[\mathrm{MPa}]}\end{array}$ & $\tan \delta$ \\
\hline $1 \mathrm{~Hz}$ & 2.6 & 0.32 & 1.8 & 0.77 & 5.6 & 0.60 & 1.8 & 1.22 \\
$10 \mathrm{~Hz}$ & 4.5 & 0.28 & 4.6 & 0.58 & 2.6 & 0.46 & 6.8 & 0.78 \\
$50 \mathrm{~Hz}$ & 6.4 & 0.16 & 10 & 0.34 & 18 & 0.26 & 15 & 0.47 \\
\hline
\end{tabular}




\section{S2 Additional FEM model details}

The characteristic size of the mesh grid under the impact zone is of $1 \mathrm{~mm}$ (element aspect ratio 1:1:1). 5 integration points through thickness were used to catch the non-linear behavior of the foam under compression. Spurious modes in the reduced (1-point) integration elements were mitigated via an hourglass viscous form ${ }^{7}$ assuring that the associated energy was below $5 \%$ of the deformation energy of the target at each step of the simulation. The gas pressure within the cell walls was assumed in the constitutive model ${ }^{8}$ to be equal to 1 bar (atmospheric pressure).

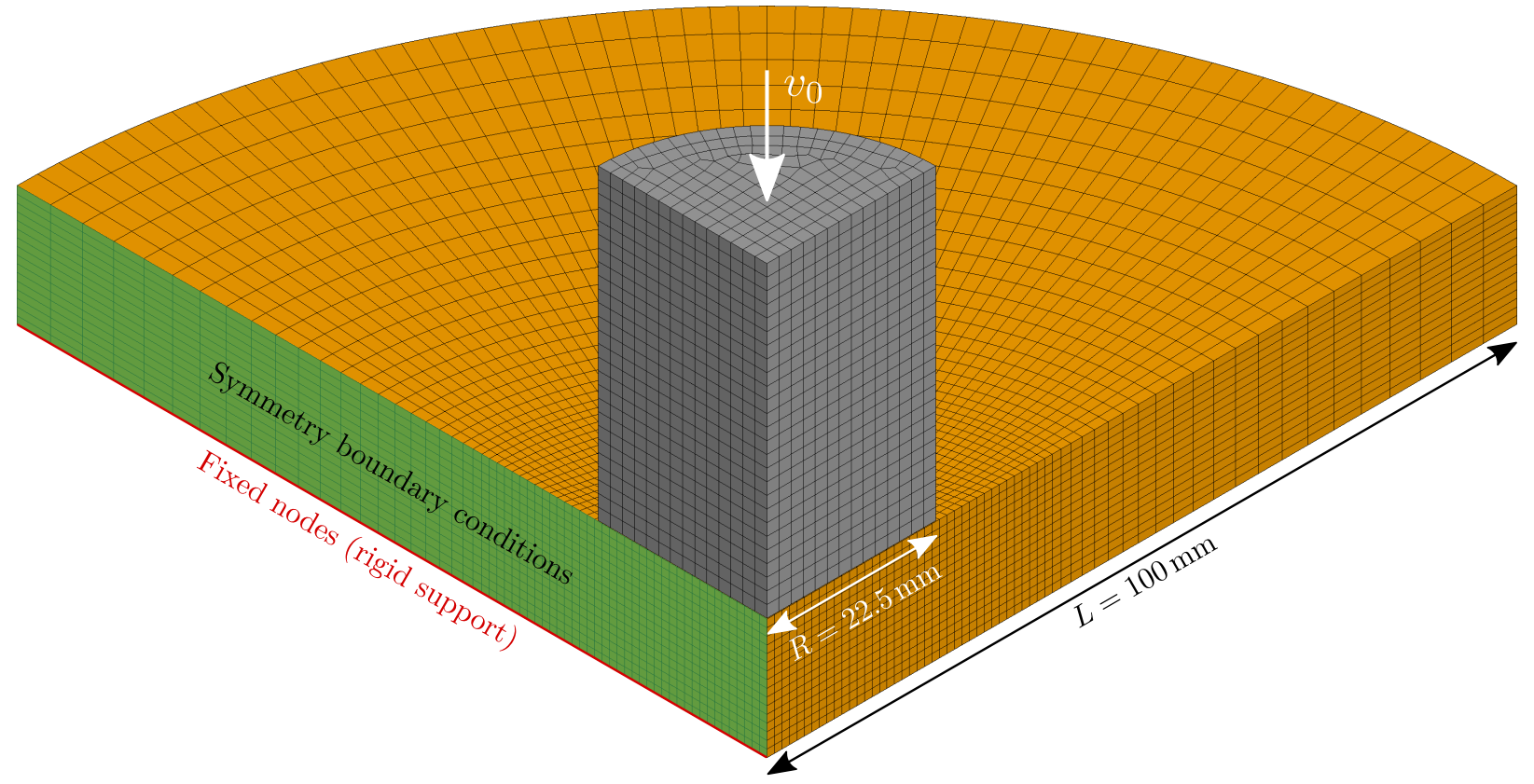

Figure S3: Schematic representation of the FEM model with geometrical characteristics and detail of the adopted boundary conditions. Same initial impact velocity $v_{0}$ and mass $m$ as in the drop weight impact test (impact energy of about $50 \mathrm{~J}$ ) were assigned to the impactor (grey part). 
Table S4: Input parameters for analytical and FEM model for the 4 protectors at $-5^{\circ} \mathrm{C}$ and $+20^{\circ} \mathrm{C}$ derived from DMTA analysis at $50 \mathrm{~Hz}$ (see ref. [1] and Supplementary Section S1.3). Critical damping coefficients $(\xi)$ are computed by Equation (3) in the main text, with $c_{\text {crit }}=2 \sqrt{k_{\mathrm{b}} m}$, where $m$ is the mass of the beam specimen used in the DMTA test.

\begin{tabular}{ccccccccccccccc}
\hline \multirow{2}{*}{$\mathbf{T}$} & \multicolumn{3}{c}{ Protector 1 } & \multicolumn{3}{c}{ Protector 2 } & \multicolumn{3}{c}{ Protector 3 } & \multicolumn{3}{c}{ Protector 4 } \\
\cline { 2 - 13 } & $\begin{array}{c}E \\
{[\mathrm{MPa}]}\end{array}$ & $\tan \delta$ & $\xi$ & $\begin{array}{c}E \\
{[\mathrm{MPa}]}\end{array}$ & $\tan \delta$ & $\xi$ & $\begin{array}{c}E \\
{[\mathrm{MPa}]}\end{array}$ & $\tan \delta$ & $\xi$ & $\begin{array}{c}E \\
{[\mathrm{MPa}]}\end{array}$ & $\tan \delta$ & $\xi$ \\
\hline$-5{ }^{\circ} \mathrm{C}$ & 13.4 & 0.039 & 0.092 & 33.4 & 0.078 & 0.192 & 28.6 & 0.050 & 0.144 & 70.4 & 0.053 & 0.190 \\
$+20^{\circ} \mathrm{C}$ & 6.4 & 0.164 & 0.269 & 10.0 & 0.339 & 0.456 & 18.0 & 0.261 & 0.594 & 15.0 & 0.472 & 0.778 \\
\hline
\end{tabular}




\section{References}

(1) Nicotra, M.; Moncalero, M.; Messori, M.; Fabbri, E.; Fiorini, M.; Colonna, M. Thermomechanical and impact properties of polymeric foams used for snow sports protective equipment. Procedia Engineer 2014, 72, 678-683.

(2) EN 1621-2, Motorcyclists' protective clothing against mechanical impact. Motorcyclists back protectors. Requirements and test methods. 2003.

(3) ISO 868, Plastics and ebonite - Determination of indentation hardness by means of a durometer (Shore hardness). 2003.

(4) ASTM D4065-12, Standard Practice for Plastics: Dynamic Mechanical Properties: Determination and Report of Procedures. 2012.

(5) Rodríguez-Pérez, M. A.; Velasco, J. I.; Arencón, D.; Almanza, O.; Saja, J. A. D. Mechanical characterization of closed-cell polyolefin foams. J Appl Polym Sci 2000, 75, 156-166.

(6) Rodríguez-Pérez, M. A. Effect of Chemical Composition, Density and Cellular Structure on the Dynamic Mechanical Response of Polyolefin Foams. Cell Polym 2002, 21, 117-136.

(7) Belytschko, T.; Ong, J. S.; Liu, W. K.; Kennedy, J. M. Hourglass control in linear and nonlinear problems. Comput Method Appl M 1984, 43, 251-276.

(8) Neilsen, M. K.; Krieg, R. D.; Schreyer, H. L. A constitutive theory for rigid polyurethane foam. Polym Eng Sci 1995, 35, 387-394. 